STUDI

FRANCESI

\section{Studi Francesi}

Rivista quadrimestrale fondata da Franco Simone

165 (LV | III) | 2011

LA RAPPRESENTAZIONE DELLA MADRE NELLA

LETTERATURA FRANCESE DEL NOVECENTO a cura di Dario Cecchetti e Michele Mastroianni

\title{
Horst F. Müller, Studien und Miszellen zu Henri Barbusse und seiner Rezeption in Deutschland
}

\section{Elisa Borghino}

\section{OpenEdition}

\section{Journals}

Edizione digitale

URL: http://journals.openedition.org/studifrancesi/5119

DOI: 10.4000/studifrancesi.5119

ISSN: 2421-5856

Editore

Rosenberg \& Sellier

Edizione cartacea

Data di pubblicazione: 1 décembre 2011

Paginazione: 671

ISSN: 0039-2944

Notizia bibliografica digitale

Elisa Borghino, «Horst F. Müller, Studien und Miszellen zu Henri Barbusse und seiner Rezeption in Deutschland», Studi Francesi [Online], 165 (LV | III) | 2011, online dal 30 novembre 2015, consultato il 14 janvier 2021. URL: http://journals.openedition.org/studifrancesi/5119; DOI: https://doi.org/10.4000/ studifrancesi.5119

Questo documento è stato generato automaticamente il 14 janvier 2021.

\section{(†) $\ominus$

Studi Francesi è distribuita con Licenza Creative Commons Attribuzione - Non commerciale - Non opere derivate 4.0 Internazionale. 


\title{
Horst F. Müller, Studien und Miszellen $z u$ Henri Barbusse und seiner Rezeption in Deutschland
}

\author{
Elisa Borghino
}

\section{NOTIZIA}

HORST F. MÜLLER, Studien und Miszellen zu Henri Barbusse und seiner Rezeption in Deutschland, Frankfurt am Main, Peter Lang, 2010, pp. 288.

1 Sulla scia di una prima bio-bibliografia pubblicata nel 2003, lo studioso e romanziere Horst F. Müller torna a presentare l'impegno letterario dello scrittore, giornalista e attivista politico francese Henri Barbusse nel corso della prima guerra mondiale.

2 Gli undici studi raccolti tra il 1975 e il 2009 costituiscono gli undici momenti principali in cui è strutturata la ricerca; il percorso mette in evidenza la partecipazione di Barbusse alla rivista «Les Marges» e studia il conseguente dibattito ruotante attorno a letteratura e politica, scaturito principalmente da una lettera di Barbusse a Montfort. Attraverso la partecipazione alla rivista non solo si instaura un dibattito sul legame esistente tra letteratura e politica, ma si esplicita inoltre la posizione presa nel corso del conflitto mondiale, con la conseguente esasperazione delle passioni fino al parossismo.

3 Di particolare rilievo la (ri)lettura del romanzo Le Feu, 1916 (Das Feuer, 1918) che, ambientato nel corso della prima guerra mondiale e spesso ridotto a semplice reportage, offre invece gli strumenti utili per un nuovo accostamento all'autore e alla sua opera.

Interessante inoltre il fatto che lo studio di Müller tenga a dimostrare come le più recenti annotazioni sul testo si scontrino con il vivo realismo per approdare ad una forte storicità del testo $\mathrm{e}$ ad una sentita dimensione mitica, puntando all'approfondimento del carattere visionario e del ruolo dell'intertestualità all'interno dell'opera. 
5 La postfazione all'edizione tedesca delle Lettres di Henri Barbusse alla moglie tra il 1914 e il 1917 e l'analisi di Briefe von der Front si rivelano altri punti chiave dello studio insieme a Stalin, 1934, costruito sulla lettera di Barbusse ad Alfred Kurella.

6 Consacrati alla diffusione dell'opera, gli ultimi tre capitoli mostrano come da un lato Barbusse sia stato accolto favorevolmente dagli espressionisti tedeschi - tra cui Yvan Goll, Kasimir Edschmid e Ludwig Rubiner-evidenziando d'altro canto come intellettuali conservatori quali Thomas Mann abbiano rifiutato Clarté, in accordo con E. R. Curtius e André Gide. L'influenza di Barbusse su Thomas Mann e soprattutto le affinità tra il romanzo L'Enfer e La montagna magica sono studiate da un punto di vista tematico e critico-analitico.

7 Risulta infine particolarmente approfondita l'analisi del cornélianisme come norma letteraria, dove la definizione di cornélianisme corrisponde alla grandezza d'animo e all'eroismo alla maniera dei personaggi di Corneille. Se da un lato Corneille è visto come una vera e propria divinità incarnata nei panni del poeta dell'eroismo, dall'altro il superamento del cornélianis-me coincide con un atto di asserzione ed una vera e propria presa di posizione letteraria. 